\title{
True Aneurysm of the Breast After Vacuum- assisted Removal of Benign Masses: A Case Report
}

This article was published in the following Dove Press journal: International Journal of General Medicine

\section{Guilin Ye \\ Wu Zhou \\ Jiawu Li \\ Wenwu Ling \\ Yan Luo}

Department of Ultrasound, West China Hospital, Sichuan University, Chengdu,

Sichuan, People's Republic of China
Correspondence: Yan Luo

Department of Ultrasound, West China

Hospital, Sichuan University, No, 37,

Guoxue Xiang, Chengdu 61004I, People's

Republic of China

Email luoyanddoc@I63.com
Rationale: The most common complications of vacuum-assisted removal (VAR) for benign breast masses are hematoma, infection, and occasionally pseudoaneurysms. To the best of our knowledge, this is the first report of a true aneurysm following VAR for breast fibroadenomas.

Case Presentation: A 50-year-old woman underwent VAR of bilateral benign breast masses under ultrasonic guidance. Routine breast ultrasound examination was performed 3 months later, and no discomfort was observed during follow-up.

Diagnoses and Interventions: Physical examination revealed a slightly palpable, arteriallike pulsation in the lateral part of the right breast. The two-dimensional ultrasound showed that there was a well-defined anechoic nodule in the right breast at the 9 o'clock position $3 \mathrm{~cm}$ from the nipple, measuring $6 \mathrm{~mm} \times 4 \mathrm{~mm}$. Color Doppler sonography demonstrated that it was a localized dilated intramammary arteriole within the colorful flow. Spectral Doppler illustrated a high-velocity turbulent arterial flow component inside. Based on these findings, the patient was diagnosed with an iatrogenic true aneurysm of the breast. Given her overall good condition, conservative treatment with regular imaging surveillance was adopted.

Outcomes: Up to now, the patient remains asymptomatic, and the size of the aneurysm has not changed.

Lessons: With the increasing use of interventional diagnosis and treatment techniques, iatrogenic vascular complications are likely to occur more frequently. Careful duplex ultrasound examination prior to or following the procedure is strongly recommended. In the absence of risk factors, we recommend a conservative approach to small, stable aneurysms. Keywords: ultrasound, breast, true aneurysm, conservative treatment

\section{Introduction}

Ultrasound-guided vacuum-assisted removal (VAR) has been widely used in the resection of benign breast masses because of its minimally invasive and aesthetic features, as well as its important role in diagnosis and treatment. Reported complications of VAR have been rare, with hematoma and infection the most common complications and occasionally pseudoaneurysms. ${ }^{1}$ To our knowledge, this report is the first case study documenting a true aneurysm of the breast after VAR. True aneurysm refers to localized dilatation of all three layers of the vessel wall: intima, media, and adventitia. It is a very uncommon nonneoplastic vascular lesion of the breast. The cause can be congenital or acquired. Only four cases of true aneurysm of 
the breast have been described in the current literatures. ${ }^{2-5}$ One case was a congenital true aneurysm of the right internal mammary artery with an arteriovenous fistula of the left breast. One case was presumed to be caused by amphetamine abuse, and the other two cases were formed for no reason. We describe a case of true aneurysm following VAR of breast fibroadenomas that was treated conservatively.

\section{Case Presentation}

A 50-year-old woman was admitted to our hospital for vacuum-assisted removal of bilateral breast fibroadenomas due to progressive growth of the masses. The patient had no history of trauma, hypertension, coagulation disorders, or any other systemic disease. She previously underwent an ultrasound-guided biopsy of bilateral breast masses, and the histological diagnosis was fibroadenomas. Ultrasoundguided VAR was performed with an 8-gauge needle under local anesthesia until the masses were no longer sonographically visible. The patient was discharged on the same day without any immediate complications.

The patient was followed up after 3 months and reported to be a good condition without any discomfort. On physical examination, the lateral part of the right breast presented mild arterial pulsation without any palpable active mass or breast bruising. The two-dimensional ultrasound showed that there was a well-defined anechoic nodule in the right breast at 9 o'clock, $3 \mathrm{~cm}$ from the nipple (Figure 1). Color Doppler ultrasonography demonstrated that it was a localized dilated intramammary artery with an intact wall, measuring $6 \mathrm{~mm} \times 4 \mathrm{~mm}$, and no evidence of fistula and surrounding thrombosis was found on repeated scans (Figure 2). Spectral Doppler displayed

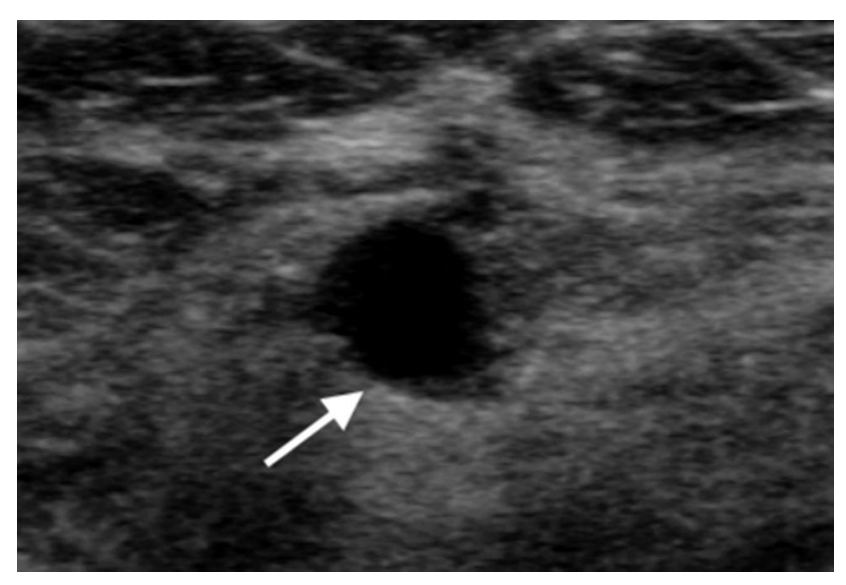

Figure I Gray-scale ultrasound showed a well-defined anechoic nodule (arrow) in the right breast.

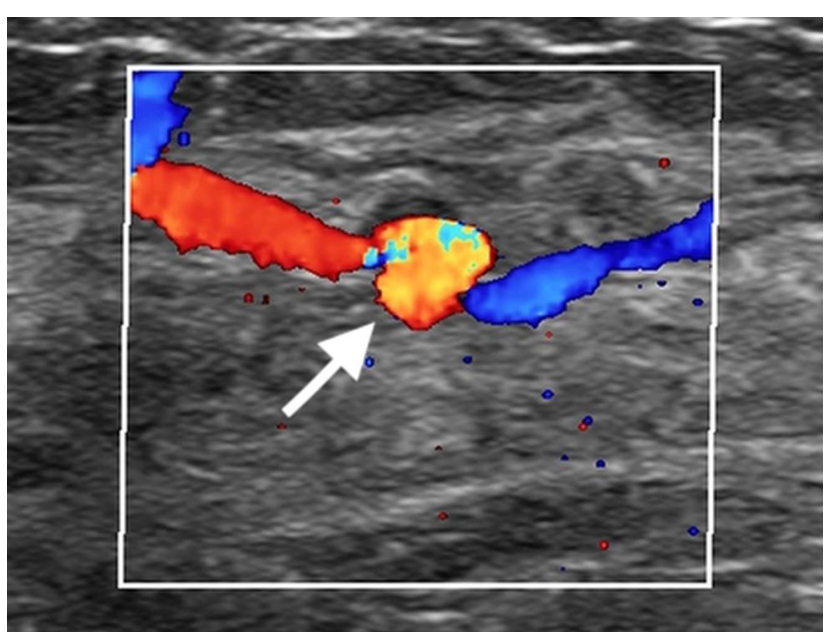

Figure 2 Color Doppler sonography illustrated that it was a localized dilated intramammary arteriole (arrow).

a high-speed turbulent arterial flow component inside (Figure 3). Power Doppler showed that blood flow was filling it in (Figure 4). The patient denied a history of trauma to the right breast or anticoagulation therapy during the follow-up visit, and no cystic structure was found in previous breast ultrasound examinations. Based on these findings, the patient was diagnosed with an iatrogenic aneurysm of the breast.

Finally, a multidisciplinary consultation was conducted regarding the patient, and a variety of management schemes were proposed for the treatment of the true aneurysm, including conservative treatment with regular imaging surveillance, surgical resection, or interventional procedures. After explaining to the patient the pros and cons of various treatment protocols, she preferred conservative treatment with regular breast ultrasound examination. The patient was also aware of the complications of the aneurysm and was told to present at a nearby hospital if there was breast pain or bruising. A recent ultrasound scan revealed no increase in aneurysm size, and she has remained asymptomatic for 18 months.

\section{Discussion}

Breast aneurysms are extremely rare. Pseudoaneurysm (PA) of the breast occurs relatively more frequently than true aneurysms. PA results from transmural disruption of an arterial wall, followed by hematoma formation, in which a tract persists between the vessel and the hematoma. ${ }^{6}$ It is thought to be more frequent in elderly people, patients with atherosclerosis, or patients on anticoagulants. However, the most common cause of PA is 


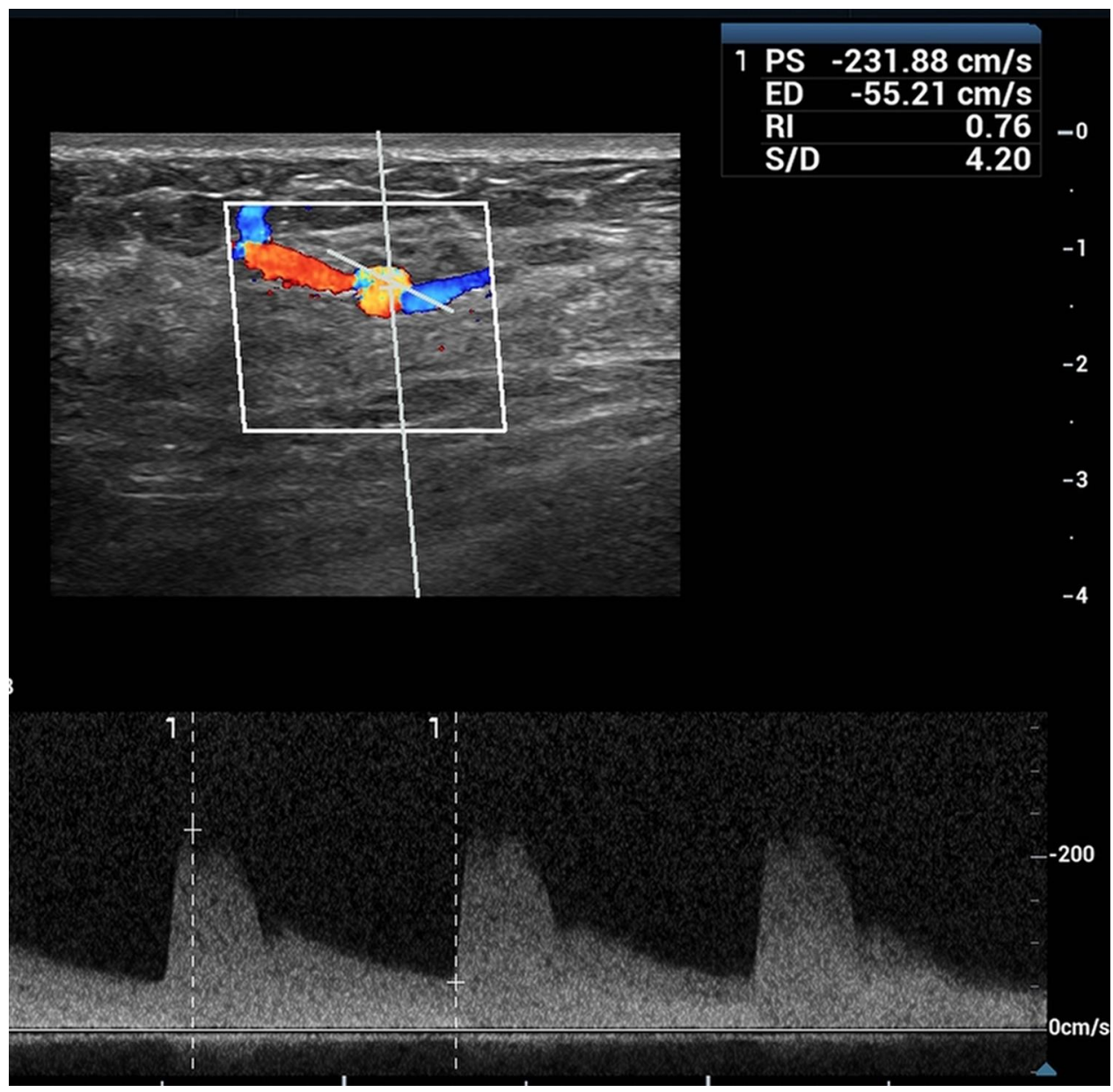

Figure 3 Spectral Doppler demonstrated a high-velocity arterial flow inside.

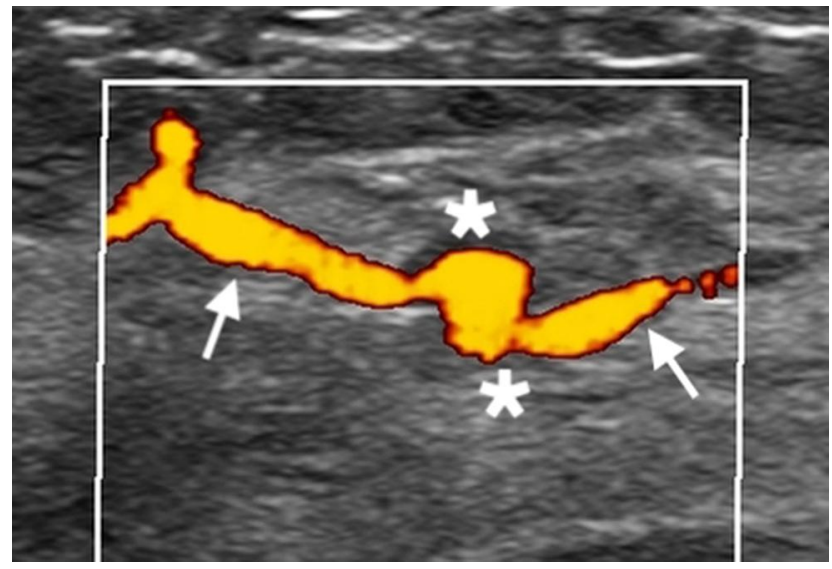

Figure 4 Power Doppler revealed that the aneurysm (asterisk) and its tract (arrow) completely filled with blood flow.

associated with trauma, a well-known complication of diagnostic interventional techniques. ${ }^{7}$ Unlike pseudoaneurysm, true aneurysms are defined as localized abnormal dilatations of all three layers of the arterial wall. The natural course of breast aneurysms is not exactly known since few cases have been reported. True aneurysms in the breast have been reported as a result of prior trauma and can appear as slowly enlarging, pulsatile masses. ${ }^{8}$ The cause of the aneurysm in our case is speculated to be related to the history of repeated interventional procedures (core needle biopsy and VAR of benign breast masses). Although the wound is very small and almost invisible to the naked eye, it is still an invasive procedure that can lead to an inflammatory response in the normal tissue around the removal target, including the blood vessel wall. In previous reports, pseudoaneurysms have often appeared as complications of interventional operations; there have been no reports of true aneurysms because puncture needles with different diameters are more likely to cause destruction of the whole vessel wall, making pseudoaneurysms easier to form. ${ }^{7}$ However, in this case, we did not find the clinical manifestations and imaging findings of a pseudoaneurysm, so this report is the first of a true breast aneurysm after VAR. 
Given the increasing prevalence of VAR, it is possible that procedure-related aneurysms could be encountered more often. The diagnosis of breast aneurysms can be suspected clinically, and Doppler ultrasonography of the breast is an ideal tool to confirm the diagnosis. ${ }^{8}$ Careful Doppler ultrasound examination prior to or following the procedure is highly recommended. For patients with clinical signs, such as extensive bleeding and hematoma during the procedure, extended use of color Doppler ultrasound monitoring in the early postoperative period might result in correct diagnosis and distinguish whether there is serious vascular damage to formulate appropriate treatment plans and provide a realistic opportunity for successful nonsurgical occlusion. True aneurysms in the breast are rare but should be distinguished from other breast masses. D'Antonio et $\mathrm{al}^{4}$ reported a case of a true aneurysm that was misdiagnosed as an intraductal papilloma, which was totally thrombosed. Due to various degrees of thrombosis, it showed a nonspecific, solid tumor-like appearance with heterogeneous hyperechogenicity or hypoechogenicity without blood flow. Atypical aneurysms with thrombi can be difficult to distinguish from pseudoaneurysms with thrombosis or intraductal tumors. Surgical resection is necessary for a final diagnosis of masses with malignant ultrasonic signs and clinical manifestations. On grayscale ultrasound, true aneurysms are similar to breast cysts or ductal dilatations. Although breast aneurysms are not common, true aneurysms can be misdiagnosed when multiple lesions are present in one breast. In our case, the aneurysm appeared as a clearbounded, anechoic cystic nodule on 2D ultrasound, which was considered to be a cyst in our first suspicion. Thereafter, the cystic structure of the breast could not be diagnosed blindly, especially in a patient with a history of breast interventional procedures, which required color flow evaluation.

Due to the limited understanding of the natural history of breast aneurysms and the paucity of experience, there is no standard management protocol known for this rare complication. It has been reported in the literature that true aneurysm of the breast has been successfully treated by surgical resection. ${ }^{4,5}$ Apart from surgical repair, percutaneous thrombin injection is also a feasible method. ${ }^{2}$ In our case, given the overall performance status of the patient (asymptomatic, no history of hypertension or anticoagulant therapy) and the small size of the aneurysm with no significant signs to be enlarged, we felt that it is safe that she be given conservative treatment with regular imaging follow-up. Further, spontaneous occlusion might be achieved through this simple monitoring without invasive intervention. ${ }^{6}$ In addition, no cases of morbidity or mortality caused by breast aneurysms have been reported in the literature. We believe that the treatment options for aneurysms should be based on the anatomical features, the risk of rupture, and the patient's comorbidities. The literature suggests that smaller fusiform aneurysms without risk factors can be treated conservatively, and changes in aneurysms can be evaluated by periodic imaging follow-up. ${ }^{9}$ For eccentric aneurysms larger than $2.5 \mathrm{~cm}$ or aneurysms with a high risk of rupture, such as in patients with refractory hypertension or long-term anticoagulation therapy, immediate surgical resection or interventional treatment is recommended. ${ }^{10}$

\section{Conclusion}

In conclusion, this study is the first report of a true breast aneurysm as a complication after VAR. With the widespread use of interventional techniques, vascular injuries related to procedures could be encountered more frequently in the future. Careful ultrasound examination before and after surgery has become particularly important. Small, stable, risk-free aneurysms can be enrolled in surveillance programs and regularly evaluated by real-time ultrasound examination.

\section{Abbreviations}

VAR, vacuum-assisted removal; PA, pseudoaneurysm.

\section{Consent Statement}

Written informed consent has been obtained from the patient for the publication of this report.

\section{Author Contributions}

All authors made substantial contributions to conception and design, acquisition of data, or analysis and interpretation of data; took part in drafting the article or revising it critically for important intellectual content; agreed to submit to the current journal; gave final approval of the version to be published; and agree to be accountable for all aspects of the work.

\section{Disclosure}

The authors have no conflicts of interest to disclose. 


\section{References}

1. Li Y, Wu Z, Yan F, et al. Pseudoaneurysm associated with arteriovenous fistula involving a superficial breast arteriole after vacuum-assisted removal of a benign mass: a case report. Medicine (Baltimore). 2018;97(36):e12250. doi:10.1097/ MD.0000000000012250

2. Cil AS, Bozkurt M, Bozkurt DK, et al. Coexistence of a congenital arteriovenous fistula of the left breast with a true aneurysm of the right internal mammary artery. Clin Med Res. 2013;11(4):237-241. doi: $10.3121 / \mathrm{cmr} .2013 .1147$

3. Cox J, Kaye B, Burn D, et al. Multiple aneurysms in the female breast: a case report. Br J Radiol. 2007;80(959):e275-e277. doi:10.1259/bjr/ 13699159

4. D'Antonio A, Addesso M, Quaranta R, et al. Intramammary aneurysm presenting as a breast mass. Breast J. 2016;22(4):466-468. doi:10.1111/tbj. 12608

5. Snow H, Cham A, Dow C, et al. True aneurysm of the breast. Breast J. 2013;19(4):444-445. doi:10.1111/tbj.12138
6. El Khoury M, Mesurolle B, Kao E, et al. Spontaneous thrombosis of pseudoaneurysm of the breast related to core biopsy. AJR Am J Roentgenol. 2007;189(6):W309-W311. doi:10.2214/AJR.05.1647

7. Dixon AM, Enion DS. Pseudoaneurysm of the breast: case study and review of literature. $\mathrm{Br} \quad J$ Radiol. 2004;77(920):694-697. doi: $10.1259 / \mathrm{bjr} / 55440225$

8. Jesinger RA, Lattin GE, Ballard EA, et al. Vascular abnormalities of the breast: arterial and venous disorders, vascular masses, and mimic lesions with radiologic-pathologic correlation. Radiographics. 2011;31(7):E117-E136. doi:10.1148/rg.317115503

9. Russell T, Creagh-Barry M. Breast pseudoaneurysm arising from core needle biopsy should be left well alone. BMJ Case Rep. 2017;2017:bcr-2017-221546. doi:10.1136/bcr-2017-221546

10. Trikha SP, Rose V, Sharma AK. An unusual origin of a breast lump: a case report. Breast J. 2002;8(6):391-392. doi:10.1046/j.15244741.2002.08410.x

\section{Publish your work in this journal}

The International Journal of General Medicine is an international, peer-reviewed open-access journal that focuses on general and internal medicine, pathogenesis, epidemiology, diagnosis, monitoring and treatment protocols. The journal is characterized by the rapid reporting of reviews, original research and clinical studies across all disease areas. The manuscript management system is completely online and includes a very quick and fair peer-review system, which is all easy to use. Visit http://www.dovepress.com/ testimonials.php to read real quotes from published authors. 